\title{
Nanoscale Strain Tomography by Scanning Precession Electron Diffraction
}

\author{
Duncan N. Johnstone ${ }^{1}$, Antonius T. J. van Helvoort ${ }^{2}$ and Paul A. Midgley ${ }^{1}$ \\ 1. Department of Materials Science and Metallurgy, University of Cambridge, 27 Charles Babbage Road, \\ Cambridge, CB3 0FS, UK. \\ 2. Department of Physics, Norwegian University of Science and Technology, NTNU, 7491 Trondheim, \\ Norway.
}

Strain within materials can be engineered on the nanoscale to control physical properties from electronics to mechanics. Methods for mapping this strain experimentally include both X-ray diffraction and electron microscopy techniques. X-ray techniques such as Laue micro-diffraction [1] and coherent X-ray diffraction [2] offer spatial resolution of $\sim 200 \mathrm{~nm}$ and $\sim 30 \mathrm{~nm}$ respectively and electron backscatter diffraction [3] reduces this only slightly. Spatial resolution down to $\sim 1 \mathrm{~nm}$ is obtained only via (scanning) transmission electron microscopy ((S)TEM) methods [4] such as geometric phase analysis, dark-field holography and scanning (precession) electron diffraction (SPED). These (S)TEM techniques have been evaluated extensively for strain mapping in semiconductor devices [4] and it has emerged that SPED offers several benefits in terms of precision, ease of implementation and balance between spatial resolution and field of view. Further, the SPED approach is highly versatile and can be applied to a wide range of materials systems and geometries.

SPED involves scanning a double-rocking electron probe [5] across the specimen and recording a PED pattern at each probe position. A four-dimensional (4D) dataset is obtained that can be analyzed in versatile post-facto computational schemes, including strain mapping. Strain maps were obtained in this work by optimizing the affine transformation, $\mathrm{D}$, that maps a reference pattern, $\mathrm{P}\left(\mathrm{x}_{0}, \mathrm{y}_{0}\right)$, onto each diffraction pattern, $\mathrm{P}(\mathrm{x}, \mathrm{y})$, in the 4D-SPED dataset. Polar decomposition of the affine matrix yields a rotation matrix, $\mathrm{R}$, and a distortion matrix, $\mathrm{F}$. The latter is easily related to strain, $\varepsilon$, in the small strain approximation (Figure 1). Here, a GaAs nanowire containing a GaAs${ }_{1-x} \mathrm{Sb}_{\mathrm{x}}$ insert was studied. The nanowire is strained due to the lattice mismatch between the Sb-rich insert and adjacent regions, providing an ideal geometry for developing methods of strain tomography. The strain distribution within these nanowires is also of significant interest in the context of optoelectronic applications owing to the modification of the local electronic band gap that it imparts.

Tomographic reconstruction of the strain component along the nanowire axis was achieved by performing a tilt series about this axis, spanning an angular range of $+-30^{\circ}$ and imposing 6-fold rotational symmetry to complete the series. At each tilt, a strain map was formed and it was assumed that the strain at each pixel these maps represents the average strain along the beam path. The component of strain along the nanowire axis was mapped at every tilt step, owing to the tilt series being performed about that axis, which enabled the reconstruction of this component as a standard scalar tomography problem. First, the "average strain" maps were converted to maps of "projected strain" by multiplication by the specimen thickness. This thickness was determined from a tomographic reconstruction performed using dark-field images formed post-facto from the SPED dataset. The projected strain maps were then used to perform tomographic reconstruction of the axial strain field via the simultaneous iterative reconstruction technique, as shown in Figure 2. 
Challenges posed by the reconstruction of the full strain tensor will be discussed, along with progress towards solving these problems to achieve tensor tomography. The precision of the methods developed and the implications of dynamical electron scattering will also be considered in detail. Particular attention will be given to the advantages obtained through the implementation of double-conical rocking in the SPED scheme as compared with scanning nano-beam electron diffraction. In summary, this work presents an initial demonstration of nanoscale strain tomography based on scanning precession electron diffraction, as well as thorough discussion of the tensor tomography problem in electron microscopy [6].

[1] F. Hofmann et al., Nature Communications, 4, (2013), p.2774.

[2] I. Robinson and R. Harder, Nature Materials, 8, (2009), p. 291-298.

[3] A. Wilkinson, G. Meaden, D.J. Dingley, Ultramicroscopy, 106, (2006), p. 307-313.

[4] D. Cooper et al., Micron, 80, (2016), p.145-165.

[5] J.S. Barnard, D.N. Johnstone and P.A. Midgley, Ultramicroscopy, 174, p.79-88.

[6] The authors acknowledge funding from the University of Cambridge, ERC grant 291522-

3DIMAGE, European Commission grant 312483-ESTEEM2 and the EPSRC NanoDTC EP/L015978/1.
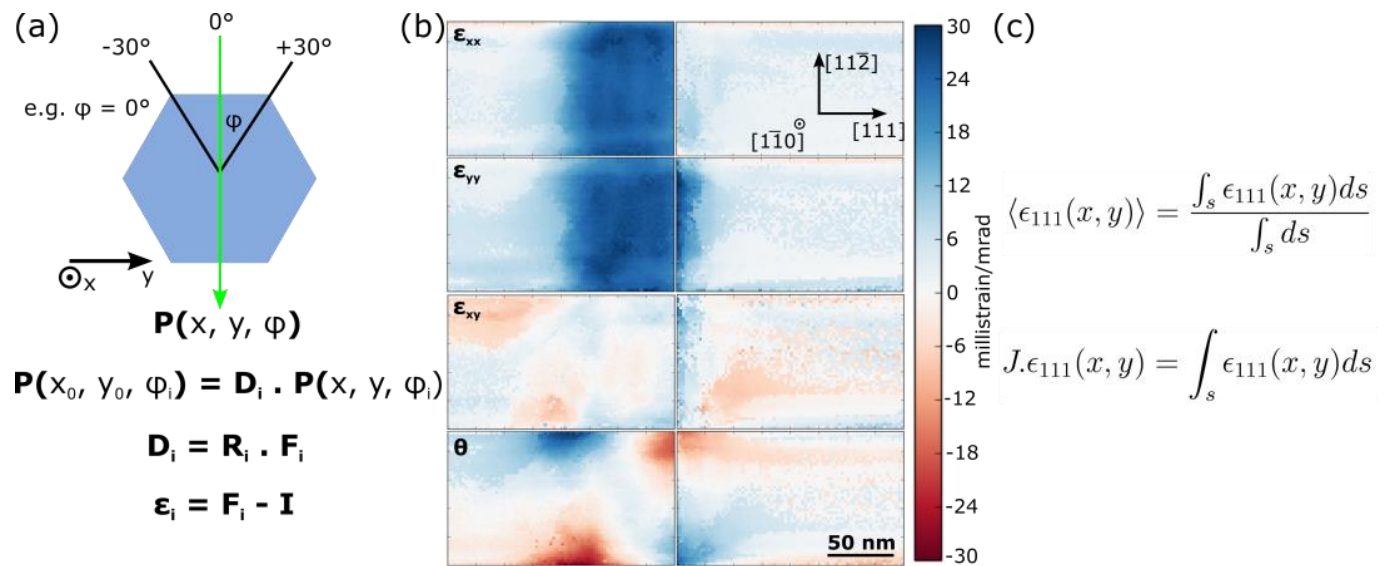

Figure 1. (a) Schematic representation of the nanowire cross section, tilt series performed and outline mathematics of strain mapping at each tilt. (b) Example strain maps obtained near to a $\langle 110\rangle$ zone axis. (c) Strain averaging along the beam path and the relationship to "projected strain", $J$.

(a)

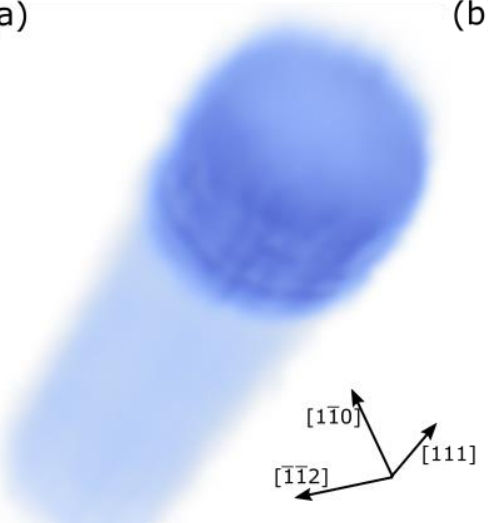

(b)

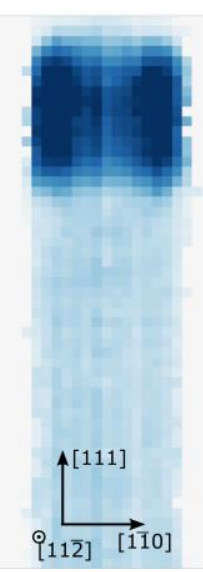

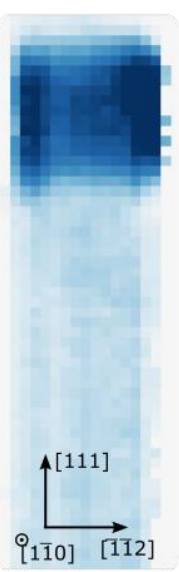

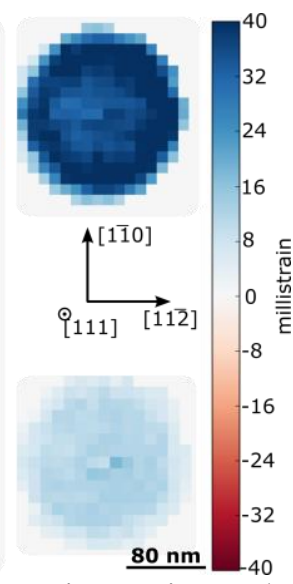

Figure 2. (a) Strain tomogram of the $\varepsilon_{111}$ component along the nanowire axis and (b) ortho-slices through the reconstruction showing variations in the strain between the center and surface of the nanowire. Note that strains are relative to unstrained GaAs so that the entire $\mathrm{GaAs}_{1-\mathrm{x}} \mathrm{Sb}_{\mathrm{x}}$ insert appears to be expanded. The hexagonal morphology can be discerned but is suppressed by the spatial resolution. 